\title{
LETTER
}

Chronic myeloproliferative neoplasms

\section{Genomic characterization of patients with polycythemia vera developing resistance to hydroxyurea}

\author{
Alberto Alvarez-Larrán ${ }^{1}$ - Alvaro Díaz-González ${ }^{2}$ Esperanza Such ${ }^{2}$ - Elvira Mora ${ }^{2}$ - Marcio Andrade-Campos ${ }^{3}$. \\ Carmen García-Hernández ${ }^{4}$ M. Teresa Gómez-Casares ${ }^{5} \cdot$ Valentín García-Gutiérrez $^{6}$ - Gonzalo Carreño-Tarragona ${ }^{7}$. \\ Marta Garrote $^{1} \cdot$ Lierni Fernández-lbarrondo $^{8}$ - José Cervera ${ }^{2} \cdot$ Beatriz Bellosillo $^{8}$ - Francisco Cervantes ${ }^{1}$. \\ Juan Carlos Hernández-Boluda $\mathbb{D}^{9} \cdot$ On behalf of the MPN Spanish Group (GEMFIN)
}

Received: 2 February 2020 / Revised: 1 April 2020 / Accepted: 23 April 2020 / Published online: 5 May 2020

(c) The Author(s) 2020. This article is published with open access

\section{To the Editor:}

Resistance/intolerance to hydroxyurea (HU) develops in $20-30 \%$ of patients with polycythemia vera (PV) and has been associated with increased risk of thrombosis, disease transformation and lower survival [1-5]. Recently, a molecular classification of myeloproliferative neoplasms (MPNs) based on data provided by next-generation sequencing (NGS) techniques has been proposed [6]. This classification, which stratifies MPN patients into eight molecular groups, may potentially be useful for personalizing prognosis and treatment; however, the impact of this genomic classification in the risk of development of $\mathrm{HU}$ resistance is unknown.

The objective of the present study was to characterize PV patients with resistance to HU by genomic classification and to assess its value in predicting disease progression and survival. Samples obtained from $61 \mathrm{HU}$-resistant patients at diagnosis $(n=38)$ and/or at time of resistance $(n=45)$ were analyzed by NGS. Cases with paired samples $(n=22)$ were sequenced at both time points. Fifty-nine HU-treated

Supplementary information The online version of this article (https:// doi.org/10.1038/s41375-020-0849-2) contains supplementary material, which is available to authorized users.

Alberto Alvarez-Larrán

aalvar@clinic.cat

1 Hematology Department, Hospital Clínic, Institut d'Investigacions Biomèdiques August Pi i Sunyer (IDIBAPS), Barcelona, Spain

2 Hematology Department, Hospital La Fe, Valencia, Spain

3 Hematology Department, Hospital del Mar, IMIM, Barcelona, Spain

4 Hematology Department, Hospital General Universitario, Alicante, Spain patients with no developed resistance were also sequenced by NGS and used as controls. Patients were hierarchically allocated into eight molecular subgroups according to the algorithm reported by Grinfeld et al. [6]. Patients were classified according to the result of the available sample or at time of resistance in those with paired samples. Resistance to HU was assessed according to the ELN modified criteria as described by Barosi et al. [7] and PV diagnosis was established according to World Health Organization criteria [8]. Informed consent was obtained for scientific use of the patients' clinico-hematological data and biological samples, and the study was approved by the Instituto de Investigación Sanitaria La Fe institutional review board. Survival analyses and time-to-event curves were calculated from date of HU initiation.

The main clinical characteristics of the $61 \mathrm{PV}$ patients with resistance to $\mathrm{HU}$ and the 59 controls are shown in supplemental Table 1. Median time on HU therapy was 4.6 years in cases and 5.9 years in controls ( $p$ not significant for the comparison). Types of resistance were need for phlebotomies $(n=13)$, progressive splenomegaly $(n=3)$, uncontrolled myeloproliferation $(n=6)$, and cytopenia $(n=39)$. All patients except one were JAK2-positive. Mutations other than $J A K 2$ were frequently observed in resistant patients, which were reclassified as PV with TP53 disruption/aneuploidy (16.4\%), PV with spliceosome/

5 Hematology Department, Hospital Doctor Negrín, Las Palmas de gran Canaria, Spain

6 Hematology Department, Hospital Ramón y Cajal, IRYCIS, Madrid, Spain

7 Hematology Department, Hospital 12 de Octubre, Madrid, Spain

8 Pathology Department, Hospital del Mar, IMIM, Barcelona, Spain

9 Hematology Department, Hospital Clínico, INCLIVA, Valencia, Spain 
Fig. 1 Distribution of mutations other than JAK2 detected by NGS in 61 hydroxyurea-resistant patients. NGS studies were performed at diagnosis (a) or at resistance (b). Group 1: TP53 disruption or aneuploidy (TP53 mutation, Chr17pLOH or Chr5-/ Chr5q-). Group 2: $\geq 1$ aberrations in chromatin or spliceosome genes $(E Z H 2, I D H 1, I D H 2$, ASXL1, PHF6, CUX1, ZRSR2, SRSF2, U2AF1, KRAS, NRAS, GNAS, CBL, Chr7/7qLOH, Chr4q/LOH, RUNX1, STAG2, and $B C O R$ ). Group 3: JAK2 homozygous mutation. Group 4: $J A K 2$ heterozygous mutation. In the central panel, each column corresponds to an individual case and colored squares denote mutated genes. The upper graph represents the number of mutations in each patient. The right graph illustrates frequency of mutated genes. All patients except one were $J A K 2$-positive. No mutation was detected in patient number 120 .
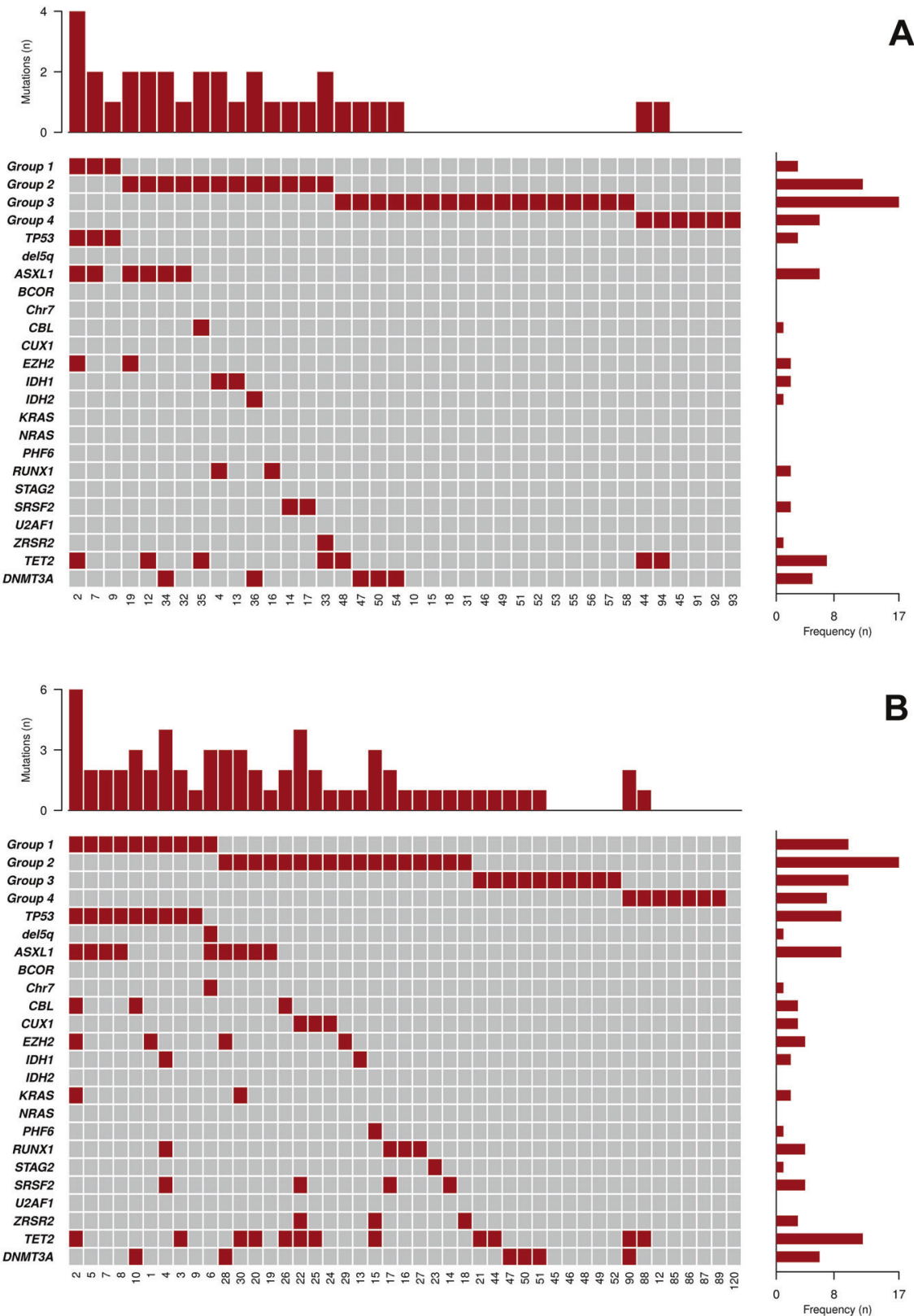

chromatin mutations (37.7\%), PV with homozygous $J A K 2$ mutation (27.9\%), and PV with heterozygous JAK2 mutation $(16.4 \%)$, whereas the corresponding figures in controls were $1.7 \%, 11.9 \%, 44 \%$, and $41 \%$, respectively $(p<0.0001$, Supplemental Table 2). Distribution of mutations by genomic classification in HU-resistant patients is shown in Fig. 1. In resistant patients with paired samples at diagnosis and at time of resistance, a total of 18 new variants were documented in 9 patients corresponding to a rate of 12.9 new mutations $\times 100$ person years. The main characteristics of these nine patients are shown in supplemental Table 3 . The genomic subgroup changed at the time of HU resistance in six cases (27\%), four of them as a result of mutation acquisition in TP53 or ZRSR2 genes (two cases each) and the remaining two due to transition from heterozygous to homozygous JAK2 mutational status.

Time to HU resistance according to genomic classification is shown in Fig. 2a. The probability of developing resistance after 5 years of HU treatment was $64 \%$ in patients with TP53 disruption/aneuploidy, $49 \%$ with spliceosome or chromatin aberrations, $27 \%$ with homozygous JAK2 mutation, and $14.5 \%$ with heterozygous JAK2 mutation ( $p<$ 0.0001 for comparison between groups). In multivariate analysis, genomic classification was associated with risk of resistance to $\mathrm{HU}$ (HR 2.2, 95\% CI: $1.5-3.2, p<0.0001$ ) after correction for age (HR: 1.01, 95\% CI: 0.97-1.03, $p=0.9$ ), sex (HR: $0.7,95 \%$ CI: $0.9-1.2, p=0.4), \mathrm{Hb}$ value (HR: $1.08,95 \%$ CI: $0.9-1.2, p=0.3$ ), leukocyte 
A
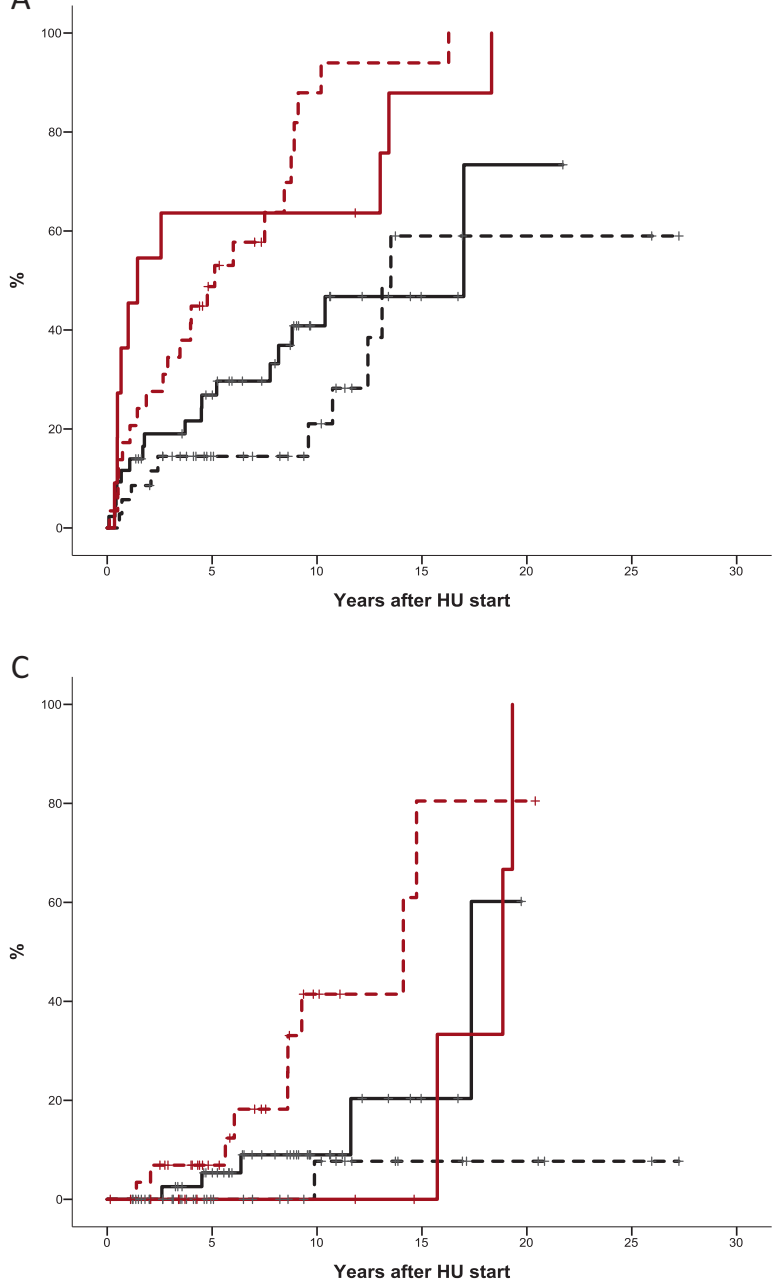

Fig. 2 Outcomes according to genomic classification in $\mathbf{1 2 0}$ patients with polycythemia vera treated with hydroxyurea (HU). a Time to hydroxyurea resistance. b Probability of survival. c Probability of myelofibrotic transformation. d Probability of progression to

count (HR: $1.1 ; 95 \%$ CI: 1.01-1.18, $p=0.02$ ), platelet count (HR: 1.0, 95\% CI: 0.9-1.002, $p=0.9$ ), and spleen size (HR 1.03, 95\% CI: $0.9-1.2, p=0.7$ ). Type of resistance according to molecular subgroup is shown in Supplemental Table 4.

With a median follow-up of 7 years from $\mathrm{HU}$ initiation (6.5 years for cases and 7.3 for controls, $p=0.6$ ) 38 patients died, resulting in a projected median survival of 15.7 years from $\mathrm{HU}$ initiation (95\% CI: 7.6-23.8). Probability of survival at 10 years from $\mathrm{HU}$ initiation was $94 \%, 75 \%, 48 \%$, and $59 \%$ in patients with heterozygous JAK2 mutation, homozygous $J A K 2$ mutation, aberrations in spliceosome/ chromatin genes, and TP53 disruption/aneuploidy, respectively (Fig. 2b, $p<0.0001$ ). In multivariate analysis and after correction for age, patients with TP53 disruption/ aneuploidy or spliceosome/chromatin mutations showed increased risk of death compared with $J A K 2$ heterozygous patients (HR: $4.2,95 \%$ CI: $1.2-15.1, p=0.026)$ and JAK2
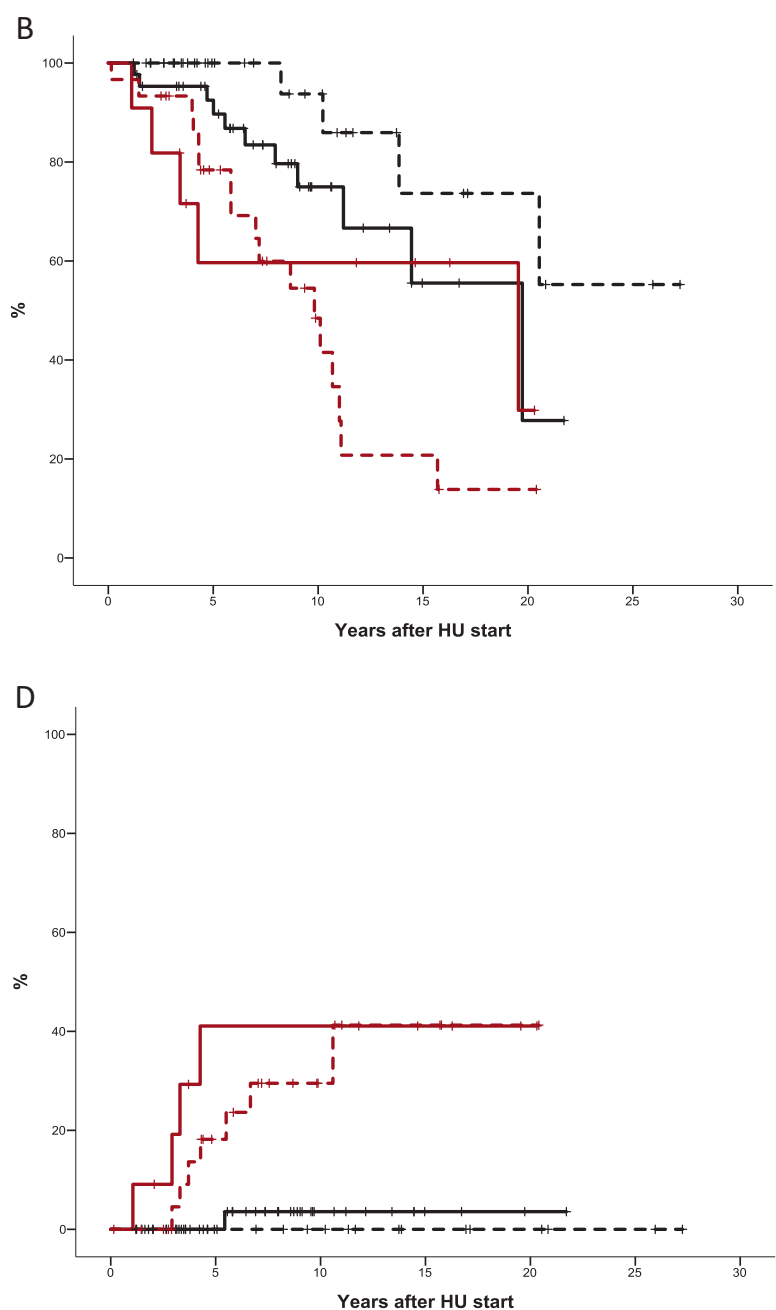

acute myeloid leukemia. Solid red line: TP53 disruption/aneuploidy. Dotted red line: spliceosome/chromatin aberration. Solid black line: homozygous $J A K 2$ mutation. Doted black line: heterozygous $J A K 2$ mutation. $p<0.0001$ for all comparisons.

homozygous patients (HR: 2.1, 95\% CI: 1.01-4.04, $p=$ 0.046).

There were no significant differences in thrombosis incidence by genomic classification except between $J A K 2$ homozygous and heterozygous patients $(p=0.047)$. Patients with spliceosome or chromatin gene aberrations progressed faster to MF than those in the other molecular groups whereas the rate of progression to MF was similar among patients with TP53 disruption/aneuploidy and JAK2 homozygosity (Fig. 2c). The probability of MF remained low only in $J A K 2$ heterozygous patients $(p<0.0001$, Fig. 2c). Nine progressions to AML and three to myelodysplastic syndrome (MDS) were registered mainly in patients with TP53 disruption/aneuploidy or aberrations in spliceosome/chromatin genes $(p<0.0001$, Fig. $2 d)$.

To the best of our knowledge, this is the largest study addressing the genomic complexity of PV patients developing resistance to $\mathrm{HU}$ and the first to apply the recently 
proposed MPN molecular classification in this clinical setting [6]. As expected, resistant patients were frequently located in the high-risk molecular groups, which would explain the high rate of disease progression and worse survival reported in such patients $[1,2]$. Thus, $17 \%$ of HUresistant patients corresponded to the TP53 disruption/ aneuploidy category, while up to $40 \%$ were classified within the group with spliceosome/chromatin gene mutations, percentages clearly higher than the ones observed in nonresistant HU-treated controls and general PV patients [6]. Moreover, in up to $27 \%$ of patients the molecular group allocated at diagnosis had changed at time of resistance, highlighting the importance of sequential studies in patients receiving cytoreductive treatment. The latter finding could be explained by the existence of subclonal mutations not detectable at diagnosis that would expand under HU therapy, or by the appearance of new mutations due to genomic instability [9-11].

HU resistance developed especially rapid in patients with TP53 mutation. Similarly, a high proportion of patients with mutations in spliceosome/chromatin genes developed resistance to $\mathrm{HU}$, but in this case the number increased progressively during follow-up, affecting virtually all patients at longest follow-up. Molecular classification revealed a particular pattern of progression under $\mathrm{HU}$ therapy in each molecular subgroup. Thus, in patients with TP53 disruption the natural history of the disease was affected mainly by the high probability of AML in the first five years of therapy, while in patients with mutations in spliceosome/chromatin genes, transformation to MF was more notable. High rates of thrombosis and progression to MF were observed in the $J A K 2$ homozygous mutation group. Finally, patients classified as heterozygous JAK2 mutation had low rates of resistance, thrombosis and disease progression during $\mathrm{HU}$ treatment.

The main limitation of the present study is the relatively small number of patients included and the lack of samples from PV diagnosis in a proportion of cases. However, it is important to underline that there were no significant differences between cases and controls regarding general characteristics such as age, sex, history of thrombosis or duration of $\mathrm{HU}$ treatment, and that the distribution of molecular groups in the controls was totally superimposable to that described in Grinfeld et al.'s pivotal study [6].

In conclusion, our data should prove useful in clinical decision-making. They support the implementation of NGS techniques in clinical practice to select the most appropriate treatment for PV patients. New drugs are clearly needed for PV patients with high molecular risk, since results of $\mathrm{HU}$ treatment are poor. Improvement of preventive measures for thrombosis is essential in HU-treated patients with homozygous JAK2 mutation. Conversely, patients classified within the heterozygous $J A K 2$ mutation category can benefit from long-term cytoreduction with $\mathrm{HU}$, as demonstrated by their low rates of resistance, thrombosis and disease progression.

Acknowledgements This work was supported by GILEAD Fellowship Program 2016 and by grants PI18/01472, PI18/00205, PI16/153 from the Instituto de Salud Carlos III (ISCIII), through the Plan Estatal de Investigación Científica y Técnica y de Innovación. We would like to thank Biobank $\mathrm{La} \mathrm{Fe}$ for providing the samples.

Author contributions AAL designed the study, collected the data, performed the statistical analysis, analyzed and interpreted the results and wrote the paper. ADG collected the data, performed the statistical analysis, analyzed and interpreted the results and wrote the paper. ES designed the study, performed the molecular studies, analyzed and interpreted the results and wrote the paper. EM, MA, CGH, MTGC, VGG, GCT, MG, LFI, and JC collected the data, interpreted the results and approved the final version. BB, FC, and JCHB interpreted the results and wrote the paper.

\section{Compliance with ethical standards}

Conflict of interest The authors declare that they have no conflict of interest.

Publisher's note Springer Nature remains neutral with regard to jurisdictional claims in published maps and institutional affiliations.

Open Access This article is licensed under a Creative Commons Attribution 4.0 International License, which permits use, sharing, adaptation, distribution and reproduction in any medium or format, as long as you give appropriate credit to the original author(s) and the source, provide a link to the Creative Commons license, and indicate if changes were made. The images or other third party material in this article are included in the article's Creative Commons license, unless indicated otherwise in a credit line to the material. If material is not included in the article's Creative Commons license and your intended use is not permitted by statutory regulation or exceeds the permitted use, you will need to obtain permission directly from the copyright holder. To view a copy of this license, visit http://creativecommons. org/licenses/by/4.0/.

\section{References}

1. Alvarez-Larrán A, Pereira A, Cervantes F, Arellano-Rodrigo E, Hernández-Boluda JC, Ferrer-Marín F, et al. Assessment and prognostic value of the European LeukemiaNet criteria for clinicohematologic response, resistance, and intolerance to hydroxyurea in polycythemia vera. Blood. 2012;119:1363-9.

2. Alvarez-Larrán A, Kerguelen A, Hernández-Boluda JC, PérezEncinas M, Ferrer-Marín F, Bárez A, et al. Frequency and prognostic value of resistance/intolerance to hydroxycarbamide in 890 patients with polycythaemia vera. Br J Haematol. 2016;172: 786-93.

3. Demuynck T, Verhoef G, Delforge M, Vandenberghe P, Devos T. Polycythemia vera and hydroxyurea resistance/intolerance: a monocentric retrospective analysis. Ann Hematol. 2019;98: 1421-6.

4. Barbui T, Tefferi A, Vannucchi AM, Passamonti F, Silver RT, Hoffman R, et al. Philadelphia-negative classical myeloproliferative neoplasms: critical concepts and management recommendations from European LeukemiaNet. Leukemia. 2018;32:1057-69. 
5. Alvarez-Larrán A, Pérez-Encinas M, Ferrer-Marín F, HernándezBoluda JC, Ramírez MJ, Martínez-López J, et al. Risk of thrombosis according to need of phlebotomies in patients with polycythemia vera treated with hydroxyurea. Haematologica. 2017;102:103-9.

6. Grinfeld J, Nangalia J, Baxter EJ, Wedge DC, Angelopoulos N, Cantrill R, et al. Classification and Personalized Prognosis in Myeloproliferative Neoplasms. N Engl J Med. 2018;379:1416-30.

7. Barosi G, Birgegard G, Finazzi G, Griesshammer M, Harrison C, Hasselbalch $\mathrm{H}$, et al. A unified definition of clinical resistance and intolerance to hydroxycarbamide in polycythaemia vera and primary myelofibrosis: results of a European LeukemiaNet (ELN) consensus process. Br J Haematol. 2010;148:961-3.

8. Arber D, Orazi A, Hasserjian R, Thiele J, Borowitz MJ, Le Beau MM, et al. The 2016 revision to the World Health Organization classification of myeloid neoplasms and acute leukemia. Blood. 2016;127:2391-405.

9. Kubesova B, Pavlova S, Malcikova J, Kabathova J, Radova L, Tom N, et al. Low-burden TP53 mutations in chronic phase of myeloproliferative neoplasms: association with age, hydroxyurea administration, disease type and JAK2 mutational status. Leukemia. 2018;32:450-61.

10. Lundberg P, Karow A, Nienhold R, Looser R, Hao-Shen H, Nissen I, et al. Clonal evolution and clinical correlates of somatic mutations in myeloproliferative neoplasms. Blood. 2014;123:2220-8.

11. Senín A, Fernández-Rodríguez C, Bellosillo B, Camacho L, Longarón R, Angona A, et al. Non-driver mutations in patients with JAK2V617F-mutated polycythemia vera or essential thrombocythemia with long-term molecular follow-up. Ann Hematol. 2018;97:443-51. 